\title{
(6) OPEN ACCESS \\ ADCOMS: a composite clinical outcome for prodromal Alzheimer's disease trials
}

\author{
Jinping Wang, ${ }^{1}$ Veronika Logovinsky, ${ }^{1}$ Suzanne B Hendrix, ${ }^{2}$ Stephanie H Stanworth, ${ }^{2}$ \\ Carlos Perdomo, ${ }^{1}$ Lu Xu, ${ }^{1}$ Shobha Dhadda, ${ }^{1}$ Ira Do, ${ }^{1}$ Martin Rabe, ${ }^{1}$ Johan Luthman, \\ Jeffrey Cummings, ${ }^{3}$ Andrew Satlin ${ }^{1}$
}

- Additional material is published online only. To view please visit the journal online (http://dx.doi.org/10.1136/ jnnp-2015-312383).

${ }^{1}$ Department of Neuroscience and General Medicine, Eisai Inc., Woodcliff Lake, New York, USA

${ }^{2}$ Pentara Corp., Salt Lake City, Utah, USA

${ }^{3}$ Cleveland Clinic Lou Ruvo Center for Brain Health, Las Vegas, Nevada, USA

\section{Correspondence to} Dr Jeffrey Cummings, Cleveland Clinic Lou Ruvo Center for Brain Health, 888 W. Bonneville Avenue, Las Vegas, NV 89106, USA; cumminj@ccf.org

Received 1 October 2015 Revised 19 February 2016 Accepted 29 February 2016 Published Online First 23 March 2016

\section{CrossMark}

To cite: Wang J, Logovinsky V, Hendrix SB, et al. I Neurol Neurosurg Psychiatry 2016;87: 993-999.

\section{ABSTRACT}

Background Development of new therapies for Alzheimer's disease (AD) is increasingly focused on more mildly affected populations, and requires new assessment and outcome strategies. Patients in early stages of $A D$ have mild cognitive decline and no, or limited, functional impairment. To respond to these assessment challenges, we developed a measurement approach based on established scale items that exhibited change in previous amnestic Mild Cognitive Impairment (aMCl) trials.

Methods Partial least squares regression with a longitudinal clinical decline model identified items from commonly used clinical scales with the highest combined sensitivity to change over time in $\mathrm{aMCl}$ and weighted these items according to their relative contribution to detecting clinical progression in patients' early stages of AD. The resultant AD Composite Score (ADCOMS) was assessed for its ability to detect treatment effect in aMCl/prodromal AD (pAD) clinical trial populations. Results ADCOMS consists of 4 Alzheimer's Disease Assessment Scale-cognitive subscale items, 2 MiniMental State Examination items, and all 6 Clinical Dementia Rating - Sum of Boxes items. ADCOMS demonstrated improved sensitivity to clinical decline over individual scales in $\mathrm{PAD}, \mathrm{aMCl}$ and in mild $\mathrm{AD}$ dementia. ADCOMS also detected treatment effects associated with the use of cholinesterase inhibitors in these populations. Improved sensitivity predicts smaller sample size requirements when ADCOMS is used in early $A D$ trials.

Conclusions ADCOMS is proposed as new standard outcome for pAD and mild AD dementia trials, and is progressing in a CAMD-sponsored qualification process for use in registration trials of $\mathrm{PAD}$.

\section{INTRODUCTION}

The pathology of Alzheimer's disease (AD) precedes the development of symptoms by many years. ${ }^{1}$ This insight has led to a shift in AD research and treatment development to earlier predementia stages of $\mathrm{AD}$, traditionally defined as amnestic mild cognitive impairment (aMCI) and, more recently, further specified as ' $\mathrm{MCI}$ due to $\mathrm{AD}$ ', or 'prodromal AD' (pAD) (as defined by the International Working Group). ${ }^{2} 3$

The earliest clinical manifestations of $\mathrm{AD}$ involve very mild decline in cognition with measurable functional impairment developing later in the disease progression. These subtle changes early in the predementia stage of $\mathrm{AD}$ are difficult to measure, and new approaches are required to detect change and establish treatment effects. Currently, there is no consensus on standard endpoints for use in aMCI populations. ${ }^{4}$ The Food and Drug Administration (FDA) has indicated that a single composite outcome may be appropriate for $\mathrm{pAD} / \mathrm{MCI}$ due to $\mathrm{AD}$ trials. ${ }^{5}$ Cognitive instruments, such as the Alzheimer's Disease Assessment Scalecognitive subscale (ADAS-cog), Mini-Mental State Examination (MMSE), and neuropsychological test items show relatively little change over time in $\mathrm{pAD} / \mathrm{aMCI}$ participants, primarily due to ceiling effects in many of the items that make up these scales. ${ }^{67}$ Scales that measure functional or global changes may be unable to capture subtle clinical decline due to the comparatively mild functional deficits in pAD/aMCI patients. ${ }^{8-10}$ While clinical tools that are widely used in $\mathrm{AD}$ dementia trials may lack overall sensitivity, certain items within these scales appear to be more responsive to clinical decline in $\mathrm{aMCI} / \mathrm{pAD}$. We sought to develop an $\mathrm{AD}$ Composite Score (ADCOMS) comprised of items from existing scales that, when combined, would be sensitive to AD-specific clinical decline in aMCI/ pAD. After identifying the items, we assessed the ability of ADCOMS to detect treatment effects in data sets from previously conducted trials of cholinesterase inhibitors with proven efficacy in $\mathrm{AD}$. The Coalition Against Major Diseases (CAMD), a component of the Critical Path Institute, ${ }^{11}$ advanced ADCOMS with the intention of establishing this approach as a qualified primary outcome measure for registration trials in $\mathrm{pAD}$.

\section{METHODS}

Data sets

Data from placebo groups, or untreated populations of four aMCI studies, were used to establish the natural progression of the condition. These data sets included the aMCI subgroup from the Alzheimer's Disease Neuroimaging Initiative (ADNI-1; ADNI-MCI, $\mathrm{n}=405$; downloaded on 20 May 2010), the placebo group from the Alzheimer's Disease Cooperative Study (ADCS) 'A randomized, double-blind, placebo-controlled trial to evaluate the safety and efficacy of vitamin $\mathrm{E}$ and donepezil HCL (Aricept) to delay clinical progression from MCI to AD' (ADCS-MCI, $n=264$ ), ${ }^{8}$ the placebo group of 'A 1 year, multicenter, randomized, double-blind, placebo-controlled evaluation of the efficacy and safety of donepezil 
Table 1 Demographics and baseline characteristics of $4 \mathrm{MCl}$ studies

\begin{tabular}{|c|c|c|c|c|c|}
\hline Study & $\begin{array}{l}\text { Placebo, } \\
\text { n }\end{array}$ & $\begin{array}{l}\text { Age, mean } \\
\text { (SD) }\end{array}$ & $\begin{array}{l}\text { Sex, male, } \\
\mathrm{n}(\%)\end{array}$ & $\begin{array}{l}\text { ApoE } \varepsilon 4 \\
\text { positive, } \\
\text { n (\%) }\end{array}$ & $\begin{array}{l}\text { CSF A } \beta \\
\text { (1-42) } \\
\text { positive, } \\
n(\%)\end{array}$ \\
\hline ADNI-MCl* & 405 & $74.2(7.41)$ & $259(64.0)$ & $\begin{array}{l}217 \\
(53.6)\end{array}$ & $\begin{array}{l}146 \\
(36.0)\end{array}$ \\
\hline ADCS-MCI & 264 & $72.3(7.54)$ & $138(52.3)$ & $\begin{array}{l}138 \\
(52.3)\end{array}$ & \\
\hline MCI412‡,§ & 388 & $69.8(10.31)$ & $223(57.5)$ & $\begin{array}{l}118 \\
(30.4)\end{array}$ & \\
\hline MCI415§, & 103 & 73.1 (6.62) & $49(47.6)$ & & \\
\hline
\end{tabular}

ApoE, apolipoprotein $\mathrm{E}$; CSF, cerebrospinal fluid; $\mathrm{MCl}$, aMCI/MCI, amnestic/mild cognitive impairment.

*Alzheimer's Disease Neuroimaging Initiative MCI data base.

†Placebo group from Eisai/Pfizer trial of vitamin E and donepezil $\mathrm{HCl}$ (Aricept) in aMCI conducted by the Alzheimer's Disease Cooperative Study (ADCS) group. ${ }^{8}$ ¥Placebo group from Eisai/Pfizer E2020-A001-412 study of donepezil in aMCI. ${ }^{9}$ §Shared data between Pfizer and Eisai.

IPlacebo group from Eisai E2020-E033-415 study of donepezil $10 \mathrm{mg} / \mathrm{day}$ in aMCI

(Clinical trials identifier: NCT00403520).

hydrochloride in patients with MCI' (MCI-412, $\mathrm{n}=388),{ }^{9}$ and the placebo group from 'Hippocampus study: comparative effect of donepezil $10 \mathrm{mg} /$ day and placebo on clinical and radiological markers' (MCI-415, $\mathrm{n}=103)^{12}$ studies. Demographic and baseline characteristics of these data sets are summarised in table 1. The pooled data set from these four trials is referred to as pooled-MCI $(\mathrm{n}=1160)$.

Two enriched populations were defined within the pooled-MCI group: cerebrospinal fluid (CSF) A $\beta 1-42$ positive (equivalent to $\mathrm{MCI}$ due to $\mathrm{AD}$ with intermediate likelihood and almost equivalent to $\mathrm{pAD}$, with exception that $\mathrm{pAD}$ criteria high CSF total tau or phosphorylated tau along with low CSF A $\beta 1-42$ levels; this population is referred to as either MCI due to $\mathrm{AD}$ or $\mathrm{pAD}$ ) and apolipoprotein $\mathrm{E}$ (ApoE) $\varepsilon 4$ carrier aMCI. ${ }^{2}$ CSF biomarkers were available only for aMCI participants in ADNI-1, of whom 146 were CSF A $1-42$ positive (A $1-$ $42 \leq 192 \mathrm{pg} / \mathrm{mL}$ ). ApoE $\varepsilon 4$ carriers totalled 473: 217 in ADNI-1, 138 in ADCS-MCI, and 118 in MCI-412. ApoE carrier status was not available for MCI-415.

In addition to the aMCI data, three mild $\mathrm{AD}$ dementia data sets were analysed to determine whether ADCOMS was equally sensitive to decline in this population. These data sets included the mild AD dementia subgroup from ADNI-1 (ADNI-mild, $\mathrm{n}=188$ ), and placebo groups from 2 ADCS clinical trials in mild $\mathrm{AD}$ dementia: 'A multicenter trial of rofecoxib and naproxen in Alzheimer's disease' $(\mathrm{n}=111){ }^{12}$ and 'VITAL-VITamins to Slow ALzheimer's Disease' $(n=170){ }^{13}$ The pooled data set using these 3 mild dementia studies is referred to as pooled-mild-AD $(\mathrm{n}=469)$.

Data sets from ADCS-MCI $(\mathrm{n}=784)^{8}$ and ' 30 -week, multicenter, randomised, double-blind, placebo-controlled evaluation of the safety and efficacy of E2020 (donepezil) in patients with Alzheimer's disease' (AD-302, $\mathrm{n}=236)^{14}$ were used to evaluate the ability of ADCOMS to detect treatment effect in aMCI and mild $\mathrm{AD}$ dementia populations treated with donepezil.

\section{Standard AD scales}

Clinical scales available for evaluation from all $4 \mathrm{aMCI}$ data sets included ADAS- $\operatorname{cog} 12$ (standard 11 items plus delayed word recall, scored 0-80), MMSE (7 items, scored 0-30), and Clinical Dementia Rating-Sum of Boxes (CDR-SB) (6 items, scored 0-18). Additional items measured in ADNI-MCI and ADCS-MCI, included traditional neuropsychological tests (15 items), Functional Activities Questionnaire (FAQ) in ADNI, ADCS-Activities of Daily Living (ADL) in ADCS-MCI (8 items that overlap between ADCS-ADL and FAQ, each scored $0-3$ ), and Neuropsychiatric Inventory (NPI; 12 items, each scored $0-12$ ). Individual items from each clinical scale for each data set included in analyses are listed in online supplementary appendix 1. MMSE and neuropsychological items were scored in reverse (ie, item maximum score minus measured score), so that, for consistency, a higher score was indicative of greater impairment on all scales.

\section{Longitudinal disease decline model in aMCI}

To apply a statistical modelling approach to characterise the relationship between disease progression and the individual items from existing $\mathrm{AD}$ clinical scales, a linear longitudinal model was constructed to characterise clinical decline. Time is an appropriate surrogate for a direct measure of clinical change over time in a progressive disorder. Because we were interested in modelling clinical decline in early $\mathrm{AD}$ stages over a relatively short period (eg, 12 months), a linear decline model provided an accurate representation of progression. The following equation represented the clinical decline from time $t_{0}$ to time $t$ (see online supplementary appendix 2):

$$
\left(t-t_{0}\right)=\sum_{i=1}^{12} d_{i} \Delta A_{i}(t)+\sum_{i=1}^{7} e_{i} \Delta B_{i}(t)+\sum_{i=1}^{6} f_{i} \Delta C_{i}(t)+\cdots
$$

where $t_{0}$ is the time at baseline, and $\Delta \mathrm{A}_{\mathrm{i}}(\mathrm{t}), \Delta \mathrm{B}_{\mathrm{i}}(\mathrm{t}), \Delta \mathrm{C}_{\mathrm{i}}(\mathrm{t})$, and so on, are change from baseline of individual scale items at time $\mathrm{t}$, corresponding to items from ADAS-cog, reversed MMSE, CDR-SB, ADL, NPI, FAQ, ADCS-ADL scale and available neuropsychological tests. The left side of Equation (1) is duration of decline (time $t-t_{0}$ ), which is the dependent variable representing rescaled clinical decline. The right side of Equation (1) is a weighted linear combination of a set of predictor variables measuring cognitive and functional decline. Full details of the mathematical derivation leading to Equation 1 can be found in online supplementary appendix 2. Equation (1) was subsequently fitted using PLS regression to identify individual clinical scale items that represent $\mathrm{AD}$-related clinical decline over time to calculate their respective weighting factors and to optimise the sensitivity of measuring clinical decline with this weighted combination.

\section{PLS regression to generate ADCOMS}

The PLS procedure (implemented by the SAS V.9.2 system, SAS Institute Inc, Cary, North Carolina, USA) was used to fit the linear decline model (Equation (1)) using longitudinal data up to 12 months. The Variable Importance of Projection (VIP) statistically summarises the contribution each variable makes to the model. Wold's criterion was used to remove items (predictor variables) with a VIP $<0.8$. The resultant composite score, ADCOMS, is a weighted linear combination of the remaining individual scale items in the final fitted PLS model using the corresponding PLS coefficients as weighting factors.

\section{Assessing sensitivity of ADCOMS to AD-related clinical decline}

The sensitivity of ADCOMS to clinical decline was measured using the mean to $\mathrm{SD}$ ratio (MSDR) of change from baseline over a fixed time period. A larger MSDR implies greater 
sensitivity (ie, greater effect size and/or less noise). The MSDR of a composite score calculated from the same data used to develop this composite score, may be inflated due to model-data dependence and must be corrected for this bias. A conservative estimate of bias due to model-data dependence was calculated to be $5.4 \%$ (data on file, Eisai Inc, and Pentara Corp).

\section{Evaluation of ADCOMS}

Comparison with existing AD clinical scales

ADCOMS was evaluated, using bootstrap methodology, by comparing its bias-adjusted MSDR for change from baseline with the MSDRs for the ADAS-cog, MMSE, and CDR-SB total scores. The bootstrap estimate of mean MSDR and its 95\% CI, were based on 10000 bootstrap samples. Assessments were conducted for the pooled-MCI data set as well as for the enriched subgroups (ApoE $\varepsilon 4$ positive and CSF A $\beta 1-42$ positive). The performance of ADCOMS was also assessed in a time frame beyond 12 months, and in populations of mild $\mathrm{AD}$ dementia.

Study sample sizes required to detect a $25 \%$ reduction in clinical decline relative to placebo after 12 months of treatment were also calculated using the bootstrap method for the pooled-MCI, pAD (CSF A $\beta 1-42$ positive), ApoE $\varepsilon 4$ carrierenriched aMCI populations, and the mild AD dementia population. Sample size calculation was based on a two-sample t test with an $\alpha$ of 0.05 (2-sided) and a statistical power of $80 \%$. Sample sizes obtained for ADCOMS were based on bias-adjusted MSDR for change from baseline.

\section{Treatment effects}

The ability of ADCOMS and other clinical scales to measure treatment effect in both aMCI and populations of mild AD dementia was evaluated by comparing donepezil and placebo groups from the ADCS-MCI and AD-302 studies, respectively. Changes from baseline in ADCOMS, and individual AD clinical scales were analysed using a mixed model for repeated measures (MMRM) approach. The MMRM model included the baseline score of the scales chosen for comparison as a covariate with treatment group, visit, and treatment group-by-visit interaction as fixed effects. A compound symmetry covariance structure was used to model the within-patient error. Least squares means, SE, and $95 \%$ CI were calculated for the within-treatment group and between treatment group differences.

\section{RESULTS}

Fitted PLS models and ADCOMS

The VIP plot for the linear clinical decline model fitted to the pooled aMCI data set using the PLS regression is shown in figure 1. The horizontal line represents the Wold's criterion for predictor deletion $(\mathrm{VIP}<0.8)$. Twelve items had VIP values $\geq 0.8$ and were included in the final model.

The final composite score, ADCOMS, was a weighted linear combination of the 12 items in the Wold's PLS model using the corresponding PLS coefficients in the fitted model (table 2). The range of ADCOMS is between 0 and 1.97. Items making contributions to ADCOMS include 4 items of the ADAS-cog; two items of the MMSE, and all six items of the CDR-SB. No additional contribution was seen with inclusion of items from other scales including neuropsychological test items, ADL/FAQ and NPI (data not shown).

When ADCOMS was applied to the pooled aMCI data set, an increase in ADCOMS value was seen between baseline (mean 0.224 , SD 0.1029; median 0.209) and 12 months (mean 0.283, SD 0.1835; median 0.247), indicating ADCOMS' ability to detect clinical decline (mean increase 0.060, SD 0.1360; median
0.033 for participants who had assessments at both baseline and 12 months). The MSDR of change from baseline was 0.4418 . The bias-corrected MSDR for change from baseline of ADCOMS was 0.4192 .

\section{Evaluation of ADCOMS}

Sensitivity to clinical decline compared with individual clinical scales

The sensitivity of ADCOMS for measuring AD-related clinical decline was compared with existing clinical scales in pooled aMCI, ApoE $\varepsilon 4$ positive and CSF A $\beta 1-42$ positive pAD/aMCI groups, and pooled-mild $\mathrm{AD}$ dementia using the bootstrap estimate of MSDR for change from baseline at 12 months. The MSDR ratios of the ADCOMS scores to individual clinical scales are presented in table 3.

Using the pooled aMCI data set, ADCOMS showed robust statistically significant improvement in sensitivity over the ADAS-cog total score (110\% (95\% CI 67\% to 201\%) improvement) and the MMSE total score (90\% (95\% CI 53\% to $152 \%$ ) improvement), and moderate, but statistically significant improvement in sensitivity over the CDR-SB (19\% (95\% CI $10 \%$ to $30 \%$ ) improvement). ADCOMS showed similar improvements over the three existing scales in the pAD/enriched aMCI subgroups. Using the aMCI APOE $\varepsilon 4$ carrier and $\mathrm{pAD} /$ aMCI CSF A $\beta 1-42$ positive data sets, ADCOMS showed 90\% (95\% CI $42 \%$ to $160 \%$ ) and $139 \%$ (95\% CI $48 \%$ to $309 \%$ ) improvement in sensitivity compared with the ADAS-cog total score, respectively; $49 \%$ (95\% CI 20\% to $88 \%$ ) and $71 \%(95 \%$ CI $26 \%$ to $138 \%$ ) improvement compared with the MMSE total score, respectively; and $21 \%$ (95\% CI $10 \%$ to $34 \%$ ) and $11 \%$ (95\% CI $-2 \%$ to $26 \%$ ) improvement compared with the CDR-SB, respectively. The improvement over the CDR-SB using the pAD/aMCI CSF A $\beta 1-42$ positive data set is significant before adjusting for bias $\{(17 \%$ (95\%CI $3 \%$ to $33 \%))\}$, but barely misses significance after adjusting for bias $\{(11 \%(95 \%$ CI $-2 \%$ to $26 \%))\}$, possibly due to using a conservative calculation for bias adjustment in ADCOMS and the much smaller data set $(n=146)$ available for analyses.

While ADCOMS was developed using longitudinal data from aMCI clinical trials up to 12 months in duration, it maintained its improvement relative to ADAS-cog, MMSE and CDR-SB up to 36 months (see online supplementary appendix 3 ).

ADCOMS was optimised for sensitivity in an aMCI population, but showed substantial improvement in sensitivity in mild AD dementia compared with the MMSE (83\% (95\% CI 50\% to $127 \%)$ improvement), and small and moderate improvements compared with the ADAS-cog (10\% (95\% CI $-7 \%$ to $29 \%)$ improvement), and the CDR-SB (19\% (95\% CI 10\% to $28 \%$ ) improvement), respectively, over 12 months.

Sample sizes required in clinical trials based on ADCOMS compared with individual clinical scales

Sample sizes required to detect a $25 \%$ reduction in clinical decline from baseline on the ADCOMS and individual scales after 12 months of treatment are shown in table 3. Sample sizes required when using the standard scales were 4.6 (95\% CI 2.8 to 9.2) times larger for ADAS-cog, 3.8 (95\% CI 2.3 to 6.4) times larger for MMSE, and 1.4 (95\% CI 1.2 to 1.7 ) times larger for CDR-SB relative to ADCOMS for the pooled aMCI population. The same trend was observed in the aMCI ApoE $\varepsilon 4$ carriers and pAD/aMCI CSF A $31-42$ positive subgroups. Sample sizes required when using the standard scales were 3.7 (95\% CI 2.0 to 6.8) and 6.3 (95\% CI 2.2 to 17.1) times larger for ADAS-cog, respectively; 2.3 (95\% CI 1.5 to 3.5 ) and 3.0 
Figure 1 Variable importance of projection plot of partial least squares model fitted using the pooled-MCI (amnestic mild cognitive impairment) data set. MMSE, Mini-Mental State Exam; ADAS-cog, Alzheimer's Disease Assessment Scale-cognitive subscale; CDR-SB, Clinical Dementia Rating, sum of boxes.

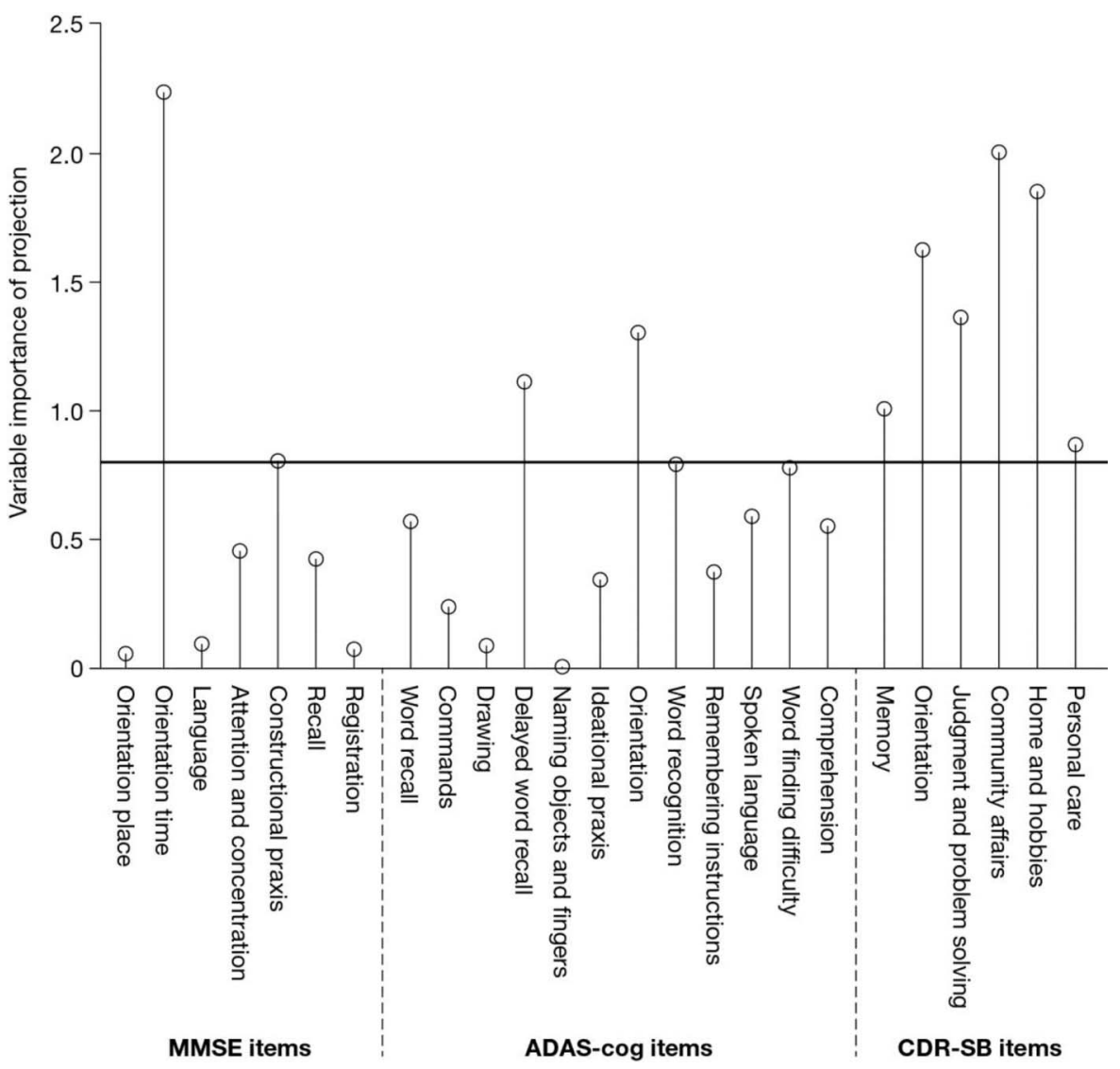

(95\% CI 1.6 to 5.4) times larger for MMSE, respectively; and $1.5(95 \%$ CI 1.2 to 1.8$)$ and $1.2(95 \%$ CI 0.97 to 1.6$)$ times larger for CDR-SB, respectively (table 3 ).

ADCOMS also required smaller sample sizes for mild AD dementia, with individual scales requiring larger sample sizes by factors of 1.2 (95\% CI 0.9 to 1.7$), 3.4$ (95\% CI 2.3 to 5.2$)$ and 1.4 (95\% CI 1.2 to 1.6) for ADAS-cog, MMSE and CDR-SB, respectively (table 3 ).

Assessing treatment effect

Treatment effects of donepezil and vitamin E compared with placebo were assessed using ADCOMS and existing scales to

Table 2 Items selected in the Wold's PLS model and their corresponding PLS coefficients

\begin{tabular}{lll}
\hline Scale & Item name & PLS coefficients \\
\hline ADAS-cog & Delayed word recall & 0.008 \\
& Orientation & 0.017 \\
& Word recognition & 0.004 \\
& Word finding difficulty & 0.016 \\
MMSE & Orientation time & 0.042 \\
& Drawing & 0.038 \\
CDR-SB & Personal care & 0.054 \\
& Community affairs & 0.109 \\
& Home and hobbies & 0.089 \\
& Judgement and problem solving & 0.069 \\
& Memory & 0.059 \\
& Orientation & 0.078 \\
\hline ADAS-cog, Alzheimer's Disease Assessment Scale-cognitive subscale; CDR-SB, Clinical \\
Dementia Rating, sum of boxes; MMSE, Mini-Mental State Exam; PLS, partial least \\
squares.
\end{tabular}

determine whether ADCOMS generated consistent outcomes and/ or improved ability to distinguish drug from placebo (table 4). Donepezil $10 \mathrm{mg}$ showed statistically significant benefit in aMCI participants (ADCS-MCI) according to ADCOMS $(p=0.019)$ and MMSE $(p=0.024)$, but not with the ADAS-cog or CDR-SB. Results using ADCOMS confirmed the lack of effect of vitamin E $(p=0.885)$, which was consistent with the results seen using individual scales $(\mathrm{p}=0.755,0.594$, and 0.419 for ADAS-cog, MMSE, and CDR-SB, respectively).

In a donepezil study in patients with mild $\mathrm{AD}$ dementia (AD-302), both donepezil 10 and $5 \mathrm{mg}$ showed improvement from baseline, while placebo showed decline from baseline. This was consistent whether measured by ADCOMS, or the three $\mathrm{AD}$ scales. Donepezil $10 \mathrm{mg}$ resulted in greater benefit than $5 \mathrm{mg}$, as assessed by all tools except MMSE. Among all the assessments, ADCOMS provided the strongest evidence $(p<0.0001)$ for treatment differences, with the benefit assessed using ADAS-cog $(p=0.0008)$, MMSE $\quad(p=0.00095)$ and CDR-SB $(p=0.017)$ not being as highly significant.

\section{DISCUSSION}

Treatments for $\mathrm{AD}$ will maintain cognitive function at higher levels if initiated early in the disease process. Clinical trials for pAD are being initiated, and improved outcomes for these new types of trials are needed. Traditional outcome scales and analytic approaches used in $\mathrm{AD}$ studies are less than optimal for measuring clinical progression in pAD/aMCI. ${ }^{15}$ ADCOMS is an outcome approach optimised to detect $\mathrm{AD}$-related clinical decline in $\mathrm{pAD} / \mathrm{aMCI}$ due to $\mathrm{AD}$. It was derived by employing a statistical model to select the most sensitive weighted combination of items from commonly used clinical scales in a large, diverse clinical data set including $\mathrm{aMCI} / \mathrm{pAD}$ populations. 
Table 3 ADCOMS improved sensitivity (MSDR) and reduced sample sizes required when compared with original scales

\begin{tabular}{|c|c|c|c|c|c|c|c|}
\hline \multirow[b]{2}{*}{ Data set $(n t)$} & \multicolumn{7}{|c|}{ MSDR* and MSDR ratio } \\
\hline & ADCOMS $\ddagger$ & ADAS-cog & Ratio§ & Reverse MMSE & Ratio§ & CDR-SB & Ratio§ \\
\hline Pooled-aMCI (963) & 0.4192 & 0.1957 & 2.1 & 0.2206 & 1.9 & 0.353 & 1.2 \\
\hline aMCI APOE ع4 carrier (402) & 0.5565 & 0.3009 & 1.8 & 0.3792 & 1.5 & 0.4629 & 1.2 \\
\hline aMCI CSF Aß1-42 positive (135) & 0.7979 & 0.3583 & 2.2 & 0.4817 & 1.7 & 0.7206 & 1.1 \\
\hline \multirow[t]{3}{*}{ Pooled-mild-AD (312) } & 0.8183 & 0.7485 & 1.1 & 0.4509 & 1.8 & 0.6887 & 1.2 \\
\hline & \multicolumn{7}{|c|}{ Sample size and sample size ratio } \\
\hline & ADCOMS $\ddagger$ & ADAS-cog & Ratioף & Reverse MMSE & Ration & CDR-SB & Ratioๆ \\
\hline Pooled-aMCI (963) & 1431 & 6549 & 4.6 & 5156 & 3.6 & 2016 & 1.4 \\
\hline aMCI APOE ع4 carrier (402) & 812 & 2774 & 3.4 & 1748 & 2.2 & 1173 & 1.4 \\
\hline aMCI CSF Aß1-42 positive (135) & 396 & 1957 & 4.9 & 1084 & 2.7 & 485 & 1.2 \\
\hline Pooled-mild-AD (312) & 376 & 449 & 1.2 & 1237 & 3.3 & 531 & 1.4 \\
\hline
\end{tabular}

The derivation of ADCOMS used robust mathematical approaches to ensure its objectivity. PLS is a method for modelling the relationship between one or more responses and several correlated independent variables. It combines a regression approach, optimising sensitivity to decline, with a technique similar to a principal components analysis which identifies item changes that converge on a unified disease construct thereby optimising specificity to AD.

ADCOMS is based on the assumption that despite the inherent heterogeneity in this slowly progressing neurodegenerative disease, a population that is sufficiently well defined to represent the early stages of $\mathrm{AD}$ will follow a similar declining path. When applied to a large aMCI population, our PLS model identified key items characteristic of the declining trajectory for $\mathrm{pAD} / \mathrm{aMCI}$.

ADCOMS demonstrates improved sensitivity to clinical decline relative to traditional $\mathrm{AD}$ scales used alone. Sensitivity to clinical decline in $\mathrm{AD}$, as measured by ADCOMS, was not further improved by adding items from other scales including ADL, FAQ, NPI and standard neuropsychological tests (data not shown). While executive function is known to decline early in aMCI, none of the corresponding neuropsychological tests included in our analyses were selected by the PLS regression procedure. CDR items may better address executive function indirectly via evaluation of changes in global/functional performance.

Table 4 Assessment of treatment effect of donepezil and vitamin E compared with placebo

\begin{tabular}{|c|c|c|c|c|c|c|c|}
\hline & & \multicolumn{3}{|c|}{ ADCS-MCI (12 months) } & \multicolumn{3}{|c|}{ Eisai-AD-302 (6 months) } \\
\hline & & Placebo & Donepezil & Vitamin E & Placebo & Donepezil & Donepezil \\
\hline Endpoint & Statistics & $(208)$ & 10 mg (182) & (208) & (71) & $5 \mathrm{mg}(74)$ & $10 \mathrm{mg}(56)$ \\
\hline \multirow[t]{4}{*}{ ADCOMS } & LSMean (SE) & $0.061(0.0080)$ & $0.034(0.0085)$ & $0.060(0.0080)$ & $0.053(0.014)$ & $-0.005(0.014)$ & $-0.033(0.016)$ \\
\hline & Difference (SE) & & $0.027(0.0117)$ & $0.002(0.0113)$ & & $0.057(0.020)$ & $0.085(0.022)$ \\
\hline & $95 \% \mathrm{Cl}$ & & 0.005 to 0.050 & -0.021 to 0.024 & & 0.018 to 0.096 & 0.043 to 0.128 \\
\hline & $\mathrm{p}$ Value & & 0.019 & 0.885 & & 0.0041 & $<0.0001$ \\
\hline \multirow[t]{4}{*}{ ADAS-cog } & LSMean (SE) & $0.79(0.309)$ & $0.08(0.328)$ & $0.93(0.311)$ & $1.6(0.552)$ & $-0.67(0.535)$ & $-1.23(0.627)$ \\
\hline & Difference (SE) & & $0.71(0.451)$ & $-0.14(0.439)$ & & $2.27(0.769)$ & $2.83(0.835)$ \\
\hline & $95 \% \mathrm{Cl}$ & & -0.18 to 1.59 & -1.00 to 0.72 & & 0.76 to 3.78 & 1.19 to 4.47 \\
\hline & p Value & & 0.116 & 0.755 & & 0.0033 & 0.0008 \\
\hline \multirow[t]{4}{*}{ MMSE } & LSMean (SE) & $-0.71(0.152)$ & $-0.20(0.163)$ & $-0.60(0.154)$ & $-0.75(0.313)$ & $0.83(0.301)$ & $0.49(0.358)$ \\
\hline & Difference (SE) & & $-0.51(0.223)$ & $-0.12(0.217)$ & & $-1.58(0.434)$ & $-1.24(0.476)$ \\
\hline & $95 \% \mathrm{Cl}$ & & -0.94 to -0.07 & -0.54 to 0.31 & & -2.43 to -0.72 & -2.17 to -0.30 \\
\hline & $p$ Value & & 0.024 & 0.594 & & 0.0003 & 0.0095 \\
\hline \multirow[t]{4}{*}{ CDR-SB } & LSMean (SE) & $0.38(0.068)$ & $0.22(0.073)$ & $0.46(0.068)$ & $0.24(0.117)$ & $-0.10(0.113)$ & $-0.19(0.134)$ \\
\hline & Difference (SE) & & $0.16(0.099)$ & $-0.08(0.096)$ & & $0.33(0.163)$ & $0.43(0.178)$ \\
\hline & $95 \% \mathrm{Cl}$ & & -0.03 to 0.36 & -0.27 to 0.11 & & 0.01 to 0.65 & 0.08 to 0.78 \\
\hline & $p$ Value & & 0.107 & 0.419 & & 0.0414 & 0.0173 \\
\hline
\end{tabular}

AD, Alzheimer's dementia; ADAS-cog, Alzheimer's Disease Assessment Scale-cognitive subscale; ADCOMS, Alzheimer's Disease clinical COMposite Score; CDR-SB, Clinical Dementia

Rating, sum of boxes; LSMean, least squares means; aMCI, amnestic mild cognitive impairment; MMSE, Mini-Mental State Exam; SE, Standard Error. 
ADCOMS was shown to be more sensitive than individual scales to AD-specific clinical decline in an enriched aMCI population defined by APOE $\varepsilon 4$ carrier status $(\mathrm{MSDR}=0.56)$ and pAD defined as aMCI with reduced CSF A $\beta 1-42$ levels (MSDR $=0.81)$. This supports the objective of developing ADCOMS for use in pAD clinical trials with disease-modifying agents. ADCOMS maintained its sensitivity in very mild AD dementia and can be applied in mixed $\mathrm{pAD} / \mathrm{mild} \mathrm{AD}$ dementia trials including pAD patients who progress to mild AD dementia over the treatment period in trials. ADCOMS' improved sensitivity to clinical decline has the benefit of reducing sample size requirements for $\mathrm{pAD}$ trials. Clinical assessment of a diseasemodifying agent targeting a $25 \%$ slowing of clinical progression in pAD patients with $80 \%$ power and two-sided $0.5 \alpha$ would require 400 patients per arm with ADCOMS, 2507 per arm with ADAS-cog, and 494 per arm with CDR-SB.

A suitable clinical outcome for clinical trials in pAD/aMCI must be sensitive to both clinical decline and treatment effect. ADCOMS demonstrated the largest sensitivity to treatment effects associated with donepezil in the ADCS-MCI trial compared with ADAS-cog, MMSE and CDR-SB. CDR-SB showed relatively high sensitivity to clinical progression in aMCI but less sensitivity to treatment effects. Overall, ADCOMS showed high sensitivity to both clinical decline and treatment effect in $\mathrm{pAD} / \mathrm{aMCI} / \mathrm{mild} \mathrm{AD}$ dementia populations. A limitation in this development approach is that changes produced by cholinesterase inhibitors may differ from effects produced by other agents.

A potential limitation of the ADCOMS is the fact that ADCOMS' performance was evaluated using the same data that were used to develop the instrument. This could inflate the performance of ADCOMS because of model-data dependence. To address this potential effect, we estimated the bias introduced by the model-data dependence and used the estimated bias to produce adjusted estimates of the ADCOMS performance. In developing ADCOMS data from ADNI, an observational study, and three interventional studies were pooled. Ideally, one would pool data only from placebo groups in interventional trials to create a new clinical outcome tool. At the present time, there exists a very limited number of $\mathrm{MCI} / \mathrm{MCI}$ due to $\mathrm{AD} / \mathrm{pAD}$ trial placebo groups that are publically available; to make analyses more robust, results from ADNI are frequently used as a simulated placebo-treated group when designing clinical trials as was done in development of ADCOMS.

To overcome these limitations, it is necessary to evaluate the performance of ADCOMS using completely independent data sets. Recently, the performance of ADCOMS in pAD participants was evaluated using an entirely independent Phase 3 data set from pAD participants. In the SCarlet RoAD (NCT01224106; WN25203) trial, $^{16}$ sensitivity to change was evaluated via MSDR for the change from baseline to Week 104 in participants randomised to placebo $(n=105)$. ADCOMS outperformed all other outcome measures, including CDR-SB, and was the only measure considered to have a robust responsiveness to decline (0.86). These results further support our conclusion that ADCOMS represents an improved clinical approach as compared with traditional $\mathrm{AD}$ tools when tracking decline in $\mathrm{pAD} / \mathrm{MCI}$ due to patients with AD.

ADCOMS is not a new clinical trial instrument. It is an analytic strategy based on commonly used clinical trial instruments that are administered in their entirety and are familiar to AD trial personnel. Implementation of ADCOMS as an outcome approach in $\mathrm{pAD} / \mathrm{aMCI} / \mathrm{mild} \mathrm{AD}$ dementia trials will be based on standard administration of conventional clinical trial instruments with analysis of the collected data, as specified by the ADCOMS methodology. ADCOMS consists of 12 items of which six are the CDR domains, five are performance-based items from ADAS-cog and MMSE; one ADAS-cog item, word finding difficulty, is judgement dependent. The six CDR items also are highly dependent on input from a caregiver and on the examiner's judgement. The composition of ADCOMS includes items from three performance-based tests-ADAS-cog, MMSE and CDR. The ADCOMS has more performance-based measures than the CDR-SB and may offer a more objective outcome assessment.

The Critical Path Institute was launched by the FDA to support activities leading to safer, faster and more efficient clinical trials. This Institute provides funding to CAMD and has the responsibility of promoting development of new clinical outcomes. The Coalition has adopted ADCOMS for qualification as a primary outcome measure in trials of $\mathrm{pAD} / \mathrm{aMCI}$.

\section{CONCLUSIONS}

Our results indicate that ADCOMS optimises sensitivity to clinical disease progression and specificity to the clinical decline that is characteristic of $\mathrm{AD}$ in the early stages. ADCOMS is more sensitive to change and treatment effects than individual trial instruments. Use of ADCOMS may enable using smaller sample sizes to demonstrate a drug-placebo difference. ADCOMS may be used as an AD clinical outcomes approach in trials of 12 months' duration or longer targeting participants with aMCI, pAD and mild AD dementia.

Contributors Substantial contributions to the conception or design of the work, or the acquisition, analysis, or interpretation of data: JW, VL, SBH and AS. Drafting of the work or revising it critically for important intellectual content: JW, VL, SBH, SHS, $C P, L X, S D, I D, M R, J L, J C$ and $A S$. Final approval of the version published: JW, VL, $S B H, S H S, C P, L X, S D, I D, M R, J L, J C$ and AS. Agreement to be accountable for all aspects of the work in ensuring that questions related to the accuracy or integrity of any part of the work are appropriately investigated and resolved: JW, VL, SBH, SHS, $C P, L X, S D, I D, M R, J L, J C$ and $A S$. No one else who fulfills the criteria above has been excluded as an author.

Funding ADNI-1 data used in the preparation of this article were obtained from the Alzheimer's Disease Neuroimaging Initiative (ADNI) data base (adni.loni.ucla. edu). ADNI-1 data collection and sharing for this project was funded by the ADNI (National Institutes of Health Grant U01 AG024904) and DOD ADNI (Department of Defense award number W81XWH-12-2-0012). ADNI is funded by the National Institute on Aging, the National Institute of Biomedical Imaging and Bioengineering, and through generous contributions from the following: Alzheimer's Association; Alzheimer's Drug Discovery Foundation; BioClinica, Inc; Biogen Idec Inc; Bristol-Myers Squibb Company; Eisai Inc; Elan Pharmaceuticals, Inc; Eli Lilly and Company; F. Hoffmann-La Roche Ltd and its affiliated company Genentech, Inc; GE Healthcare; Innogenetics, NV; IXICO; Janssen Alzheimer Immunotherapy Research \& Development, LLC.; Johnson \& Johnson Pharmaceutical Research \& Development LLC.; Medpace, Inc; Merck \& Co., Inc; Meso Scale Diagnostics, LLC; NeuroRx Research; Novartis Pharmaceuticals Corporation; Pfizer Inc; Piramal Imaging; Servier; Synarc Inc; and Takeda Pharmaceutical Company. The Canadian Institutes of Health Research is providing funds to support ADNI clinical sites in Canada. Private sector contributions to ADNI are facilitated by the Foundation for the National Institutes of Health (http://www.fnih.org). The grantee organisation is the Northern California Institute for Research and Education, and the study is coordinated by the Alzheimer's Disease Cooperative Study at the University of California, San Diego. ADNI data are disseminated by the Laboratory for Neuro Imaging at the University of California, Los Angeles. This research was also supported by NIH grants P30 AG010129, K01 AG030514, and the Dana Foundation.

Competing interests JW, VL, CP, LX, SD, ID, MR, JL and $A S$ are employees of Eisai Inc. SBH is a consultant to Eisai and has received compensation from them for this work. SHH and CP are employees of SBH. JLC has received, in kind, research support from Avid Radiopharmaceuticals and Teva Pharmaceuticals; JLC has provided consultation to Abbvie, Acadia, ADAMAS, Alzheon, Anavex, Avanir, Biogen-Idec, Biotie, Boehinger-Ingelheim, Chase, Eisai, Forum, Genentech, Grifols, Intracellular Therapies, Lilly, Lundbeck, Merck, Neurotrope, Novartis, Nutricia, Otsuka, QR Pharma, Resverlogix, Roche, Suven, Takeda and Toyoma companies; JLC had provided consultation to GE Healthcare and MedAvante; JLC owns stock in ADAMAS, Prana, Sonexa, MedAvante, Neurotrax and Neurokos; JLC owns the copyright of the Neuropsychiatric Inventory; JLC has provided expert witness consultation regarding olanzapine and ropinerol. 
Provenance and peer review Not commissioned; externally peer reviewed.

Open Access This is an Open Access article distributed in accordance with the Creative Commons Attribution Non Commercial (CC BY-NC 4.0) license, which permits others to distribute, remix, adapt, build upon this work non-commercially, and license their derivative works on different terms, provided the original work is properly cited and the use is non-commercial. See: http://creativecommons.org/ licenses/by-nc/4.0/

\section{REFERENCES}

1 Sperling RA, Aisen PS, Beckett LA, et al. Toward defining the preclinical stages of Alzheimer's disease: recommendations from The National Institute on Aging-Alzheimer's Association workgroups on diagnostic guidelines for Alzheimer's disease. Alzheimers Dement 2011;7:280-92.

2 Albert MS, DeKosky ST, Dickson D, et al. The diagnosis of mild cognitive impairment due to Alzheimer's disease: recommendations from the National Institute on Aging-Alzheimer's Association workgroups on diagnostic guidelines for Alzheimer's disease. Alzheimers Dement 2011;7:270-9.

3 Dubois B, Feldman HH, Jacova C, et al. Revising the definition of Alzheimer's disease: a new lexicon. Lancet Neurol 2010;9:1118-27.

4 Hampel H, Frank R, Broich $\mathrm{K}$ et al. Biomarkers for Alzheimer's disease: academic, industry and regulatory perspectives. Nat Rev Drug Discov 2010;9:560-74.

5 U.S. Food and Drug Administration. Guidance for industry Alzheimer's disease: developing drugs for the treatment of early stage disease. Washington DC, 2013.

6 Diniz BS, Yassuda MS, Nunes PV, et al. Mini-mental State Examination performance in mild cognitive impairment subtypes. Int Psychogeriatr 2007;19:647-56.
7 Cano SJ, Posner HB, Moline ML, et al. The ADAS-cog in Alzheimer's disease clinical trials: psychometric evaluation of the sum and its parts. I Neurol Neurosurg Psychiatry 2010;81:1363-8.

8 Petersen RC, Thomas RG, Grundman M, et al. Vitamin E and donepezil for the treatment of mild cognitive impairment. N Engl J Med 2005;352:2379-88.

9 Doody RS, Ferris SH, Salloway S, et al. Donepezil treatment of patients with MCl: a 48-week randomized, placebo-controlled trial. Neurology 2009;72:1555-61.

10 Thal L, Ferris SH, Kirby L, et al. A randomized, double-blind, study of rofecoxib in patients with mild cognitive impairment. Neuropsychopharmacology 2005;30:1204-15.

11 Romero K, de Mars M, Frank D, et al. The Coalition Against Major Diseases: developing tools for an integrated drug development process for Alzheimer's and Parkinson's diseases. Clin Pharmacol Ther 2009;86:365-7.

12 Jelic V, Kivipelto $M$, Winblad B. Clinical trials in mild cognitive impairment: lessons for the future. J Neurol Neurosurg Psychiatry 2006;77:429-38.

13 Aisen PS, Schneider LS, Sano M, et al. High-dose B vitamin supplementation and cognitive decline in Alzheimer disease: a randomized controlled trial. JAMA 2008;300:1774-83.

14 Rogers SL, Farlow MR, Doody RS, et al. A 24-week, double-blind, placebo-controlled trial of donepezil in patients with Alzheimer's disease. Donepezil Study Group. Neurology 1998;50:136-45.

15 Harrison J, Minassian SL, Jenkins L, et al. A neuropsychological test battery for use in Alzheimer disease clinical trials. Arch Neurol 2007;64:1323-9.

16 Lasser R, Ostrowitzki S, Scheltens $P$, et al. Efficacy and safety of gantenerumab from the phase 3 SCarlet RoAD trial, a study of ganenerumab in patients with prodromal AD. J Prev Alzheimer Dis 2015;2:275-6. 